\title{
Evolved A-type stars
}

\author{
M. Parthasarathy \\ Indian Institute of Astrophysics, Bangalore - 560034, India \\ email: partha@iiap.res.in
}

Abstract. A review of various types of evolved A stars is presented.

Keywords. Stars: abundances, stars: evolution, stars: post-AGB, binaries: general, stars: mass loss, circumstellar-matter

\section{Introduction}

Low and intermediate mass stars and massive stars during their post-Main Sequence evolution go through the evolved A-type star stages. The evolved A-type stars are located in the H-R diagram on the horizontal branch (HB), post-HB, post-asymptotic giant branch (post-AGB), and also in the region occupied by supergiants. Detailed multiwavelength studies of various types of evolved A-type stars enables us to understand the nucleosynthesis, mixing, diffusion, mass-loss, and similar processes, as well as the evolutionary sequence/links with their cooler and hotter analogues.

\section{A-type post-AGB stars}

Post-AGB stars (proto-planetary nebulae (PPNe)) are objects that have recently evolved off the AGB (after the termination of the AGB phase of evolution of low and intermediate mass stars) but have not yet reached high enough temperatures to photoionize their circumstellar envelopes. This evolutionary stage is very short-lived depending on the core mass (Schönberner 1983, Blöcker 1995). The low and intermediate mass stars evolve through the post-AGB phase and planetary nebulae $(\mathrm{PNe})$ phase and end as white dwarfs. During the transition from the tip of the AGB to the PN phase they appear as A-type post-AGB stars for a short period.

That some bright A and F supergiants have far-infrared (IRAS) colours similar to $\mathrm{PNe}$ and detached cold circumstellar dust shells similar to PNe central stars was first pointed out by Parthasarathy \& Pottasch (1986). They concluded that these stars are not massive supergiants, but low mass stars in the post-AGB stage of evolution. From further analyses and multi-wavelength observations of IRAS sources with far-IR colours similar to PNe, several post-AGB stars were discovered (Parthasarathy \& Pottasch 1986, 1989, Lamers et al. 1986, Hrivnak et al. 1989, Kwok 1993 and references therein, Van Winckel 2003 and references therein). They have supergiant spectral types from cool K, G, F, A types to OB types, forming an evolutionary sequence in the transition region from the tip of the AGB to early stages of PN (Parthasarathy 1993a, Parthasarathy et al. 1993, 1995). Some are high galactic latitude A and F supergiants with low metallicities and high radial velocities. It is now believed that many high galactic latitude $A$ and $F$ type supergiants are old low-mass stars in the post-AGB stage of evolution (Parthasarathy \& Pottasch 1986, Lamers et al. 1986, Bond \& Luck 1987). The AGB phase of evolution of these stars was terminated only recently by the superwind type of severe mass-loss which removed most of the outer envelope. Because of the very thin extended outer envelope that remained around the core these post-AGB stars mimic the spectra of supergiants. 


\subsection{Very metal-poor post-AGB A-supergiants}

Recent studies have established the existence of a class of post-AGB A-supergiants with extremely low iron-group abundances $(\mathrm{HR} 4049([\mathrm{Fe} / \mathrm{H}]=-4.8$, Waelkens et al. 1991, Takeda et al. 2002), HD 44179 (central star of Red Rectangle) $([\mathrm{Fe} / \mathrm{H}]=-3.1$, Waelkens et al. 1996), HD $52961\left([\mathrm{Fe} / \mathrm{H}]=-4.8\right.$, Waelkens et al. 1991), $\mathrm{BD}+39^{\circ} 4926([\mathrm{Fe} / \mathrm{H}]=$ -2.9). Their photospheric abundances are similar to the gas-phase abundances of the interstellar medium. The refractory elements $\mathrm{Fe}, \mathrm{Mg}, \mathrm{Si}, \mathrm{Al}, \mathrm{Ti}, \mathrm{Ca}$, etc are depleted and the abundances of volatile elements C, N, O, S, and Zn do not show such extreme depletions. The photospheric abundances of these stars correlate with the grain condensation temperatures (Bond 1991, Van Winckel et al. 1992, Parthasarathy et al. 1992). Van Winckel et al. (1992), Takeda et al. (2002), and Waelkens et al. (1996) found the Zn abundances in the extremely Fe-poor post-AGB stars HD 52961, HR 4049 and HD 44179 to be : HD $52961([\mathrm{Zn} / \mathrm{H}]=-1.5,[\mathrm{Fe} / \mathrm{H}]=-4.8), \mathrm{HR} 4049([\mathrm{Zn} / \mathrm{H}]=-1.3,[\mathrm{Fe} / \mathrm{H}]=-4.7)$, and $\mathrm{HD} 44179([\mathrm{Zn} / \mathrm{H}]=-0.6,[\mathrm{Fe} / \mathrm{H}]=-3.3) . \mathrm{S}$ and $\mathrm{Zn}$ have very low condensation temperatures and therefore do not easily condense into dust grains. From Figure 6 and Table 2 in Takeda et al. (2002) it is clear that the abundance of $\mathrm{Zn}$ traces the original metallicity of stars that show the depletion of refractory elements.

The nearly normal abundances of $\mathrm{Zn}, \mathrm{S}, \mathrm{C}, \mathrm{N}, \mathrm{O}$ and the extreme depletions of refractory elements $\mathrm{Fe}, \mathrm{Mg}, \mathrm{Si}, \mathrm{Al}, \mathrm{Ti}, \mathrm{Ca}$, etc. in the very metal-poor A-type post-AGB supergiants indicate that the depletion of the refractory elements is the result of chemical fractination. The presence of circumstellar dust shells/disks around these stars lends strong support to the idea that the depleted refractory elements are in circumstellar dust grains. Bond (1991) suggested that there is a selective removal of the refractory elements from the photosphere through grain formation and mass-loss. It is still not understood how the dust and the gas are separated resulting in the remarkable peculiarity in the photospheric abundances of very metal-poor A-type post-AGB stars.

The processes that produce photospheres with depleted refractory elements in the $\lambda$ Boo stars and in the A-type post-AGB stars described above may be the same (Parthasarathy 1994).

\subsection{Binarity of very metal-poor A-type post-AGB stars}

There is conclusive evidence that all the known very metal-poor post-AGB A and F supergiants (HR 4049, HD 44179, HD 52961, HD 46703, BD +39 4926, and HD 213985) with depleted refractory elements in their photospheric abundances are single-lined spectroscopic binaries with orbital periods of several hundred days (Van Winckel et al. 1995). Waters et al. (1992) suggested that the gas-dust separation needed to account for the depletion pattern and photospheric abundance peculiarities, the presence of binary companions (Van Winckel et al. 1995), and dust disks (Cohen et al. 2004, Van Winckel et al. 2000) may be a necessary condition. For HR 4049 Monier \& Parthasarathy (1999) found that its UV energy distribution is very deficient compared to that of the standard A6 Ib star. An ATLAS 9 model $\left(T_{\text {eff }}=7500 \mathrm{~K}, \log g=2.0\right.$ and $\left.[\mathrm{Fe} / \mathrm{H}]=-5.0\right)$ best fits the optical continuum. The UV-flux deficiency of HR 4049 scales as $\lambda^{-1}$ (Monier \& Parthasarathy 1999, Waelkens et al. 1995). The UV and optical light variations of HR 4049 are found to occur in phase as a result of variable circumstellar extinction (Monier \& Parthasarathy 1999, Waelkens et al. 1991). The high galactic latitude (b = $57^{\circ}$ ) post-AGB star HD 213985 (A2 Ia) also shows variable optical and UV circumstellar extinction (Waelkens et al. 1987, 1995, Ambika \& Parthasarathy 2004, Whitelock et al. 1989). Further study of the optical and UV variability of the A-type post-AGB stars HR 4049 and HD 213985 and similar stars may enable us to understand the nature of the circumstellar dust around the evolved A-type stars and the depletion of refractory 
elements. However, there are several A and F type post-AGB stars with dust disks (Sivarani 2000), but without any depletion of refractory elements in their chemical composition. For example HD 101584 which is a late A or early F post-AGB supergiant (Sivarani et al. 1999) with a hot and cold circumstellar dust disk and bipolar outflow does not show any depletion of refractory elements in its photospheric spectrum (Sivarani 2000, Sivarani et al. 1999).

The A-type post-AGB binary HD 44179 (Red Rectangle) (Cohen et al. 2004) is associated with a bipolar protoplanetary nebula with biconical appearance known as the Red Rectangle. High resolution HST images reveal a dusty disk and complex structures. Many are unique to this object. The Red Rectangle and the central A-type post-AGB star are unique spectroscopically. Its spectrum shows a plethora of unidentified optical, molecular bands in emission (Van Winckel et al. 2002) superimposed on a continuous Extended Red Emission (ERE) (Cohen et al. 1975, Witt \& Boroson 1990). The carrier of the ERE is still under investigation (Witt \&Vijh 2004). Schultheis et al. (2002) found two or three $\mathrm{A}$ and B stars with mid-IR excesses whose optical spectra indicate the presence of ERE. Further study of these stars is needed. These stars may be similar to HD 44179. HD 44179 (Red Rectangle) is one of the brightest known sources of extended red emission as well as of unidentified infrared (UIR) band emission which is attributed to PAHs. Recently Vijh et al. (2004) discovered a band of blue luminescence in the Red Rectangle nebula. They suggest that the spectrum of this newly discovered blue luminescence is most likely fluorescence from small neutral polycyclic aromatic hydrocarbon (PAH) molecules. Some other A and F-type post-AGB stars also show PAHs and dusty disks. Some of the best examples are HD 44179, CRL 2688, Hen 401, and Hen 1475 (Cohen et al. 2004, Sahai 2001, Sahai et al. 1999, Waelkens et al. 1996, Parthasarathy et al. 2001a).

\section{F-type post-AGB stars with overabundance of carbon and s-process elements}

Post-AGB stars which have gone through the carbon-star stage on the AGB are expected to show the products of the triple-alpha process, CN and ON cycling and the s-process on the surface as a result of thermally pulsing AGB evolution and the third dredge-up. Chemical composition analyses of several slightly metal-poor $([\mathrm{Fe} / \mathrm{H}]=-0.5)$ F-type post-AGB stars with $21 \mu \mathrm{m}$ IR emission features show that they are all overabundant in carbon and s-process elements (Parthasarathy et al. 1992, Van Winckel 2003, Reddy et al. 1997, 2002, Reyniers et al. 2004). These post-AGB stars show a clear relation between neutron irradiation and the s-process overabundances based on the ratio of barium peak elements (heavy s-process elements) to strontiumr-peak elements (light s-process elements).

From a comparison with the predictions of the recent AGB models, Reyniers et al. (2004) suggest that the observed spread in nucleosynthesis efficiency is intrinsic and indicates that different ${ }^{13} \mathrm{C}$ pockets are needed for stars with comparable mass and metallicity to explain their abundances.

Several post-AGB stars lack overabundances of s-process elements. Their photospheres and circumstellar dust shell are found to be oxygen rich indicating the absence of efficient third dredge-up in these stars. All the post-AGB stars with $21 \mu \mathrm{m}$ emission are found to show high $\mathrm{C} / \mathrm{O}$ ratios and overabundances of s-process elements. These stars have evolved through carbon star stage on the AGB (Parthasarathy 1999, 2000).

Some of the most s-process enriched F-type post-AGB stars are IRAS $05341+0852$ $([\mathrm{Fe} / \mathrm{H}]=-0.7,([\mathrm{~s}-$ process $/ \mathrm{Fe}]=+2.2$, Reddy et al. $(1997)$, and IRAS 06530-0213 $([\mathrm{Fe} / \mathrm{H}]=-0.5,[\mathrm{~s}$-process $/ \mathrm{Fe}]=+2.06$, Reyniers et al. $(2004)$. The optical spectra of 
these two stars are completely dominated by atomic transitions of s-process elements. For low-metallicity stars $([\mathrm{Fe} / \mathrm{H}]<-1)$ the s-process nucleosynthesis can lead to large overabundances of lead $(\mathrm{Pb})$ with respect to other s-process elements such as barium (Ba). Low-metallicity $([\mathrm{Fe} / \mathrm{H}]<-1)$ A and $\mathrm{F}$ type post-AGB stars with overabundances of carbon and s-process elements are expected to show overabundances of lead $(\mathrm{Pb})$. Discovery and study of such stars will enable us to further understand the s-process nucleosynthesis as well as the lead $(\mathrm{Pb})$ rich binary Blue Metal-Poor stars (BMP stars) (Preston \& Sneden 2000, 2005, Sneden et al. 2003). The binary companions (now unseen) of the lead rich Blue Metal-Poor stars seem to have gone through a post-AGB stage similar to the F-type post-AGB stars with overabundances of carbon and s-process elements. The Binary Blue Metal-Poor stars seems to have acquired their overabundances of carbon and s-process elements including lead $(\mathrm{Pb})$ via mass transfer during the AGB and post-AGB evolution of their companion stars (Preston \& Sneden 2005, Sneden et al. 2003, Sivarani et al. 2004).

\section{Hot post-AGB stars}

The A-type post-AGB stars evolve towards the left in the HR-diagram at constant luminosity into hotter post-AGB stars. As a result of photoionisation of the surrounding circumstellar dust and gas shell by the hot post-AGB star, they eventually appear as planetary nebulae with hot post-AGB central stars. From the analysis of IRAS data and spectra of IRAS sources with far-IR colours similar to post-AGB stars and PNe several new hot post-AGB stars have been identified (Parthasarathy et al. 2000a, Parthasarathy et al. 2001b, Parthasarathy \& Pottasch 1989). Gauba \& Parthasarathy (2003) studied the UV(IUE) spectra of several high galactic latitude hot post-AGB stars. In many cases, the UV(IUE) spectra suggested partial obscuration of the hot central stars due to circumstellar dust. The circumstellar extinction law in these cases was found to be linear in $\lambda^{-1}$ suggesting an upper limit of $\sim 200 \AA$ as the radii of the small grains. Carbon-rich and oxygen-rich features were identified in the ISO spectra of these objects (Gauba \& Parthasarathy 2004). Absorption lines of C II, N II, O II, Al III, Si III and Fe III and emission lines of Hi, Hei, Cir, Ni, [Nir], Or, [O I], Mgir, Alir, SiII, Vi, Mni, Fe III, [FeII], and [CrII] have been identified in the high resolution spectra of some hot post-AGB stars (Parthasarathy et al. 2000b, Klochkova et al. 2002, Gauba et al. 2004). P-Cygni profiles of $\mathrm{H}_{\alpha}$ indicate ongoing post-AGB mass loss in these stars. The hot post-AGB star, IRAS01005+7910 (Klochkova et al. 2002) was found to be carbon-rich. Gauba et al. (2004) estimated C/O 0.78 for IRAS13266-5551.

Several hot post-AGB stars have also been identified in the high galactic latitudes and the halo of our Galaxy on the basis of their derived atmospheric parameters and chemical composition (Conlon et al. 1991, 1994, McCausland et al. 1992, Moehler \& Heber 1998, Mooney et al. 2002 and references therein). (These stars appear to be hotter analogues of A and F - type post-AGB stars). These stars seem to have evolved from the G, F, A - type post-AGB stars in that order. However, the abundance patterns of hot post-AGB stars found in the halo of our Galaxy show significant differences when compared with the abundance pattern of G, F, A - type post-AGB stars. Most significant are the underabundances of carbon in the halo hot post-AGB stars. Such carbon underabundances are also observed in the hot-post AGB stars in the globular clusters. Thus it is likely that the hot post-AGB stars with underabundances of carbon left the AGB before undergoing the third dredge-up.

The observed abundance patterns of post-AGB stars shows the following groups : (1) Post-AGB A and F supergiants with nearly normal abundance of Fe and slightly 
overabundant CNO and oxygen-rich circumstellar dust shells. The high galactic latitude F-supergiant HD 161796 (Parthasarathy \& Pottasch 1986) is a good example of this group, (2) A-type post-AGB supergiants showing extreme underabundance of Fe and other refractory elements, and nearly normal abundances of CNO, S and Zn. Stars belonging to this group are HR 4049, HD 52961, and HD 44179 (Van Winckel et al. 1992, 1995, Van Winckel 2003, Takeda et al. 2002), (3) F-type post-AGB supergiants are carbon-rich and overabundant in s-process elements indicating that they have gone through the third dredge-up during their AGB stage of evolution. IRAS $05341+0852$ is a good example of such a post-AGB star (Reddy et al. 1997), and (4) The high galactic latitude low gravity hot post-AGB supergiants show underabundances of Fe and significant underabundances of carbon indicating that these are old disc or halo stars in the post-AGB stage of evolution (McCausland et al. 1992, Parthasarathy et al. 2000b, Mooney et al. 2002). Further studies are needed to understand how the AGB and post-AGB evolution of low and intermediate mass stars result in the above mentioned post-AGB abundance patterns.

\section{Rapidly evolving A and B type post-AGB stars}

From multiwavelength studies of IRAS sources with far-IR (IRAS) colours similar to PNe few rapidly evolving A and B type post-AGB stars have been discovered (Parthasarathy et al. 1993, 1995, 2000b, 2001b, Bobrowsky et al. 1998, Gauba \& Parthasarathy 2003). The best examples of rapidly evolving A and B type post-AGB stars are Henize $1357=$ IRAS $17119-5926=$ SAO $244567=$ Stingray Nebula and SAO $85766=$ IRAS $18062+2410$. Less than 100 years ago they were A type post-AGB stars and now they are OB central stars of low excitation planetary nebulae.

\subsection{Henize 1357 = Stingray Nebula}

Hen 1357 was the first object that was shown to have evolved from a B1 type post-AGB supergiant into a PN in the extremely short timescale of 20 years (Parthasarathy et al. 1993, 1995, Bobrowsky et al. 1998). The UV (IUE) spectra of Hen 1357 (see Figure 1 in Parthasarathy et al. 2001b), shows the rapid evolution of the central star from 1988 to 1996. The P-Cygni profiles of $\mathrm{NV}(1240 \AA)$ and $\mathrm{C} \operatorname{IV}(1550 \AA)$ in the spectra taken in 1988 , had indicated a terminal wind velocity of $-3500 \mathrm{~km} \mathrm{~s}^{-1}$. The wind appears to have completely ceased by 1994 (Fiebelman 1995, Parthasarathy et al. 1995). We find that the object has faded by a factor of 3 in the UV, from 1988 to 1996. Then, if the luminosity were to remain the same, the temperature of the central star should have increased from $37000 \mathrm{~K}$ to $50000 \mathrm{~K}$ in the same period. The fading of the UV continuum and the termination of the stellar wind (see Figure 1 in Parthasarathy et al. 2001b) suggest that the nuclear fuel is almost extinct as a result of the post-AGB mass loss. Alternatively, the fading could be due an episode of high mass-loss and it is possible that the shell hydrogen burning has not stopped or stopped temporarily. The fading suggests that the central star of Hen 1357 is rapidly evolving into a DA white dwarf.

The rapid evolution of Hen 1357 indicates that the fading is occurring much more rapidly than predicted, considering that it has taken 2700 years to go from the tip of the AGB to $T_{\text {eff }}=21000 \mathrm{~K}$ ( $T_{\text {eff }}$ in 1971 ), and about 20 years to turn into a PN. If the nuclear shell burning has stopped, it has stopped at a much lower surface temperature than any of the post-AGB evolutionary models suggest. Using a distance of $5.6 \mathrm{kpc}$, Parthasarathy et al. (1993) estimated the luminosity of the central star to be $3000 L_{\odot}$. The core mass luminosity relation then suggests that the core mass should be $0.55 M_{\odot}$. A $0.6 M_{\odot}$ core mass post-AGB star takes about 10,000 years to evolve from the tip of the AGB to the white-dwarf cooling track. The luminosity, the core mass, observed rapid evolution and 
fading of of Hen 1357 are not in agreement with the time scales of evolution of low or high mass post-AGB models. Recent HST images of Hen 1357 (Bobrowsky et al. 1998) showed the presence of a $1.67^{\prime \prime} \times 0.92^{\prime \prime}$ nebula around the central star. They also revealed the presence of collimated outflows and a binary companion to the central star of Hen 1357. The object needs to be monitored in the UV, the optical and the IR during the next few decades to understand the birth and early evolution of PNe and their central stars.

\section{2. $S A 085766$}

SAO85766 appears to have rapidly evolved in the last 75 years (Arkhipova et al. 1999, Parthasarathy et al. 2000b) similar to Hen 1357 described above. It was classified as a high galactic latitude $\left(\mathrm{b}=20^{\circ}\right)$, post-AGB star having far infrared colors similar to PNe (Volk \& Kwok 1989, Parthasarathy 1993b). In the HDE catalogue it was classified as an A5 star based on a plate taken in July 1924 (JD 2423971) (Arkhipova et al. 1999). Now it is a B1 supergiant with a low excitation nebula (Arkhipova et al. 1999, 2001a, Parthasarathy et al. 2000b). Its A5 spectral type in 1924 indicates that its $T_{\text {eff }}$ in 1924 was $8500 \mathrm{~K}$. Now its B1 I type spectrum and an analysis of recent high resolution spectra yields $T_{\text {eff }}=22000 \mathrm{~K}$. The UV colors of this star listed in the catalogue of stellar ultraviolet fluxes indicate that in 1973 its spectral type was around A5 I (Parthasarathy et al. 2000b). However, the spectral energy distribution of this star obtained in 1985-87 indicated that it is an early B-type star (B1 I). An analysis of its high resolution spectra revealed underabundances of carbon and metals, a high radial velocity, and the presence of low excitation nebular emission lines (Parthasarathy et al. 2000b). These characteristics coupled with its high galactic latitude, the presence of a PN type cold detached shell, and the variations observed in its spectrum suggest that SAO85766 has evolved rapidly during the last 75 years from a A5 type post-AGB star and it is now in early stages of the PN phase as a B1I type post-AGB star. The significant underabundance of carbon indicates that SAO 85766 left the AGB before undergoing third dredge-up (Parthasarathy et al. 2000b, Mooney et al. 2002, Ryans et al. 2003). SAO 85766 shows irregular rapid light variations of low amplitude due to pulsation (Arkhipova et al. 1999, 2001a). In the UV similar light variations are detected (Gauba \& Parthasarathy 2003).

According to Blöcker (1995) a post-AGB star of $0.7 M_{\odot}$ traverses the spectral interval from $\mathrm{F}$ type to $\mathrm{B}$ type in about 100 years. The rapid post-AGB evolution of Hen 1357 and SAO 85766 indicates that their core-masses are of the order of $0.7 M_{\odot}$. About 100 years ago these two stars were A, F type post-AGB supergiants. Post-AGB mass-loss may have speeded their evolution into hot-post-AGB and PN stages.

\section{3. $L S I I+3426$ and $L S I V-12111$}

LS II + 3426 (IRAS20462+3416 = V1853 Cyg) was classified as a massive B1.5 Ia - Iabe supergiant by Turner \& Drilling (1984). They estimated its distance to be $17.8 \mathrm{kpc}$ by assuming that it is a massive population I B supergiant located in the outer edge of the Galaxy. Parthasarathy (1993a) discovered it to be an IRAS source with far-IR (IRAS) colours similar to A and F-type post-AGB supergiants and PNe. Based on its far infrared colors, global energy distribution, low gravity, and high galactic latitude, Parthasarathy (1993a) suggested that LS II +34 26 (IRAS20462+3416) is a low-mass post-AGB B-type supergiant surrounded by a cold detached dust envelope located at a distance of 3 to 4.6 kpc, rather than a massive population B supergiant. The B supergiant spectrum of LS II +3426 shows an evolutionary sequence of post-AGB supergiants from cooler $(\mathrm{G}, \mathrm{F}, \mathrm{A})$ to hotter (B and O) types. The nebular emission lines in the optical spectrum of LS II+34 26 indicated that it is rapidly evolving into a very low excitation PN (Parthasarathy 1994, 
Garcia-Lario et al. 1997). Such nebular emission lines seems to absent in the spectrum of LS II + 3426 obtained by Turner \& Drilling in 1977. Differences in the spectrograms obtained by Turner \& Drilling in 1977 and 1981 and the recent spectra of LS II +34 26 (Parthasarathy 1994, Garcia-Lario et al. 1997, Arkhipova et al. 2001b) suggest that it is rapidly evolving into the PN stage. A multiwavelength study by García-Lario et al. (1997) confirmed that the central star is surrounded by a very low excitation and compact nebula (Ueta et al. 2000). The presence of P-Cygni profiles indicate ongoing post-AGB mass-loss (Garcia-Lario et al. 1997, Arkhipova et al. 2001b). Like Hen 1357 and SAO 85766, LS II +34 26 seems to be another rapidly evolving hot post-AGB star. Recently Arkhipova et al. (2001b) made photometric and spectroscopic observations of LSII +34 26 and found rapid irregular low amplitude light variations similar to that of SAO 85766 (Arkhipova et al. 1999, 2001a).

LS IV -12 111 (IRAS 19590-1249) was classified as a high galactic latitude hot postAGB star based on its IRAS data and high galactic latitude (Parthasarathy 1990a, 1993b). Analysis of its high resolution spectrum by McCausland et al. (1992) and Conlon et al. (1993) confirmed that it is a hot post-AGB star rapidly evolving into a low excitation planetary nebula. McCausland et al. (1992) derived chemical compositions of several high latitude hot post-AGB stars. In contrast to the cooler post-AGB stars, LS IV -12 111 showed a severe carbon depletion (Ryans et al. 2003). Similar carbon depletions have been reported in other hot post-AGB stars at high galactic latitudes. These include LS II+34 26 (García Lario et al. 1997), PG1323-086, PG1704+222 (Moehler \& Heber 1998) and SAO85766 (Parthasarathy et al. 2000b).

\section{Final helium shell flash objects}

Some post-AGB stars which are entering the white dwarf cooling track (pre-white dwarfs) with enough helium in their envelopes may experience a final helium shell flash (Schönberner 1979, Iben 1982). As a result of this late thermal pulse the very hot postAGB star returns to the AGB. The transition is characterized by an increase in luminosity and a drop in effective temperature in a very short time (of the order of 5 to 15 years). During this transition the photosphere becomes very rich in helium, C, N, O and sprocess elements. Herwig (2001) has shown that the evolutionary time scale of very late thermal pulse stars strongly depends on the value chosen for the convective mixing efficiency parameter. The evolutionary paths have a double loop structure (see Figures 1, 2, and 3 in Lawlor \& MacDonald 2002). The double loop in the HR diagram is a result of the penetration of the He-flash driven convection zone into H-rich layers. During this evolutionary phase the star once again very rapidly passes through the effective temperature and luminosity region occupied by the A and F type post-AGB stars.

Sakurai's object (V4334 Sgr) (Duerbeck et al. 2000), FG Sge (Blöcker \& Schönberner 1997), and V605 Aql (Harrison 1996) are understood to be the objects that experienced very late thermal pulse (final helium shell flash). All three have increased in luminosity, decreased in temperature, and show evidence for significant changes in chemical composition. From detailed study and monitoring of these stars, we may be able to test stellar evolution and nucleosynthesis models of the very late thermal pulse which occurs in about $10 \%$ of the central stars of planetary nebulae (Iben \& MacDonald 1995). V4334 Sgr (Sakurai's object) evolved very rapidly from a pre-white dwarf stage to the AGB in less than six years (Duerbeck et al. 2000). Lawlor \& MacDonald (2002) indicate that each of the three observed objects (Sakurai's object, V605 Aql, and FG Sge) represent one of the three crossings of the HR diagram from hot to cool (Sakurai's object), from cool to hot (V $605 \mathrm{Aql}$ ) and then back to cool giant (FG Sge). During these crossings of 
the HR diagram these stars appear to have spent a very brief time as A-F post-AGB stars for the second time or third time. These three objects may once again pass through the A-F post-AGB stars stage within the next 10 to 50 years. Lawlor \& MacDonald 2002) find that the critical aspect of the double loop evolution (their Figures 1, 2, and 3) is the requirement that the convective mixing efficiency be much lower than predicted by standard mixing length theory.

A very late thermal pulse (final helium shell flash) is also used to explain the [WC] and [WC]-PG 1159 central stars of planetary nebulae (Acker \& Neiner 2003, Parthasarathy \& 1998). The F-type post-AGB stars (see Section 3) with overabundances of carbon and s-process elements as they evolve towards higher temperatures may become [WC] Central stars of planetary nebulae (Parthasarathy 1999).

\section{Unusual eruptive and nova-like variables}

Recentlya few unusual eruptive variables have been discovered whose evolutionary stages and progenitors are not clear. These are V838 Monocerotis, V4332 Sagittari, and V445 Puppis. It is unclear if they are experiencing late thermal pulses (final helium shell flash), or nova like outbursts and if they are evolved massive stars or low mass stars belonging to a new class of objects.

V838 Mon was first reported to be in outburst in 2002 and became very bright $(V=$ 6.7 ) and blue and became a very red object. The light of the outbursts illuminated the circumstellar matter, resulting in spectacular HST images of the expanding light echo (Bond et al. 2003). It now has cooled and shows a late M spectral type to become the first supergiant classified as type L. The observations indicate that V838 Mon has evolved to a cool temperature of the order of $2500 \mathrm{~K}$ (Banerjee \& Ashok 2002) within 130 days after the outburst. Recent studies of the circumstellar envelope and infrared data indicate that V83 Mon underwent two or three prior outbursts in the past. It may be a post-AGB star or an evolved giant going through repeated outbursts and mass-loss episodes. The progenitor of V838 Mon may be an A-type star.

Two other stars that had nova-like outbursts, brightening and rapid cooling similar to V838 Mon are V4332 Sagittari (Martini et al. 1999, Munari et al. 2002, Banerjee et al. 2003) and V445 Puppis (Ashok \& Banerjee 2003 and references therein). The pre and post-outburst flux distribution of V445 Puppis appears to correspond to that of F and A stars (Ashok \& Banerjee 2003). The nova-like outbursts of V838 Mon, V4332 Sagittari and V445 Puppis may or may not be related to late thermal pulse/final helium shell flash of post-AGB stars. These stars may be close binaries with evolved companions and accretion and mass-transfer events. Further spectroscopic and photometric monitoring and detailed chemical composition analyses of V838 Mon, V 4332 Sagittari and V 445 Puppis and similar objects are needed to understand if they are related to the post-AGB evolution of single low or intermediate mass stars.

\section{A-type post-HB and post-AGB stars in globular clusters and related UV-bright stars in globular clusters}

There are several types of evolved A-type stars in globular clusters including blue straggler stars, interacting binaries, A and B type horizontal branch (HB) stars (Moehler 2005), A-type post-HB and post-AGB stars (Ambika et al. 2004, Jasniewicz et al. 2004, Mooney et al. 2004). In earlier surveys some were classified as UV-bright stars (Zinn et al. 1972). The term UV-bright stars was introduced by Zinn et al. (1972) for stars in globular clusters that are above the horizontal branch (HB) and bluer than red giants. Further studies showed that the UV-bright stars in globular clusters include suprahorizontal 
branch stars, post-AGB stars, post-early-AGB stars and AGB-manque stars (de Boer 1985, 1987, Dorman et al. 1993, Moehler 2001). The list of UV-bright stars in globular clusters prepared by Zinn et al. (1972), Harris et al. (1983), and de Boer (1987) contain several A-type post-AGB, post-HB and post-early-AGB (PEAGB) stars.

Chemical composition studies of B, A, and F type UV-bright stars in globular clusters reveal that some of them are post-AGB A and F stars showing evidence for third dredgeup and overabundance of carbon and s-process elements for example ROA 24 in $\omega$ Cen (Gonzalez \& Wallerstein 1994), while some show severe carbon deficiency indicating that they left the AGB before the third dredgeup occurred for example ZNG 1 in M10, ROA 5701 in $\omega$ Cen and Barnard 29 in M13 (Moehler et al. 1998, Jasniewicz et al. 2004, Mooney et al. 2004).

Recently Ambika et al. (2004), Jasniewicz et al. (2004), and Mooney et al. (2004) derived chemical composition of few A and B type evolved stars in several globular clusters. Some are in the post-HB and the post-AGB stages of evolution. The abundance pattern in a few A-type post-HB stars indicates the presence of diffusion and radiative levitation process, already found in blue-HB (BHB) stars in globular clusters (Moehler 2005). Chemical composition studies of several types of evolved stars and UV-bright stars in globular clusters are needed to further understand their evolutionary sequences and links and abundance peculiarities.

\section{Blue stragglers}

Globular clusters contain exotic evolved A and B stars that cannot be explained by canonical stellar evolutionary models, for example, the blue straggler stars. Blue stragglers are present in all the globular clusters. The explanations for their formation involve mass transfer in and/or the merger of a binary star system, or collisions between the stars. All these mechanisms may be at work in the globular clusters resulting in the formation of blue stragglers. Recent Hubble Space Telescope observations of a large sample of globular clusters reveal that every globular cluster contains between 40 and 400 blue stragglers (Piotto et al. 2002). The population does not correlate with either stellar collision rate (as would be expected if all blue stragglers were formed via collisions) or total mass (as would be expected if all blue stragglers were formed via the unhindered evolution of a subset of the stellar population) (Davies et al. 2004). The observed blue straggler frequency in globular clusters can be the result of the combination of the two formation processes, i.e., collisions or mergers in a crowded place (dynamical blue stragglers) and the evolution of isolated binaries (primordial blue stragglers). The comparison of the observed blue straggler distribution in 47 Tuc (Mapelli et al. 2004) with the simulated distribution supports the hypothesis that internal blue stragglers result from stellar collisions, while external (outside of cluster 20 core radii) are exclusively generated by mass transfer in primordial binaries (where are evolved in isolation experiencing mass transfer). Recent observations of a few blue stragglers in globular clusters indicate the presence of circumstellar shells around them (De Marco et al. 2004).

In wide binaries blue straggler stars may result when the primary evolves off the Main Sequence transferring mass on to the Main Sequence secondary. The blue stragglers and blue metal-poor binary stars (Preston \& Sneden 2005) in the galactic halo may have resulted from such a process (Sneden et al. 2003).

A number of blue stragglers in old stellar systems are found to be photometrically variable (Mateo 1993). Some are pulsating blue stragglers and others eclipsing binary blue stragglers. Detailed spectroscopic and photometric study of photometrically variable blue stragglers (pulsating and eclipsing) can provide the physical properties of blue stragglers 
and can help us to understand the formation of blue stragglers in globular clusters, in old open clusters, and in the field. Some photometrically variable blue stragglers are dwarf Cepheids, or SX Phoenicis variables. The reason for the relative lack of large-amplitude pulsating blue stragglers in old open clusters is unclear.

\section{Close-binary systems with A-type components}

There are many Algol-type close binaries in which the primary stars are of A-spectral type. The Algol-type systems are semi-detached binaries. The cool subgiant/giant secondaries have filled their Roche lobes, which still show evidence of mass-transfer and mass-loss. The present A-type primaries are not in thermal equilibrium. The rapidlyoscillating (pulsating) and mass-accreting A-type components of Algol-type eclipsing binary systems form a small group (Mkrtichian et al. 2004). These are Y Cam, AB Cas, RZ Cas, R CMa, TW Dra, AS Eri, RX Hya, and AB Per. Except for R CMa whose primary is a F1V star in all other above mentioned systems the pulsating primary components are A-stars. The observed pulsation periods range from 22 minutes to 282 minutes. The Atype stars in Algol-type close-binaries are important for asteroseismology as they permit asteroseismic estimates of accretion rates (Mkrtichian et al. 2004) and for understanding the mass-loss, the mass-transfer and the evolution.

Recently Mkrtichian et al. (2004) studied the frequency spectrum of the rapidly-oscillating mass-accreting A3 V component of AS Eri. AS Eri is a semi-detached eclipsing binary with an orbital period of 2.66 days. The $\mathrm{A} 3 \mathrm{~V}\left(1.92 M_{\odot}, 1.8 R_{\odot}\right)$ primary is well within its Roche lobe. The cool very low mass secondary $\left(0.207 M_{\odot}, 2.25 R_{\odot}\right.$ subgiant fills its Roche lobe. AS Eri evolved to the present configuration with a substantial loss of angular momentum. There are rapid pulsations in the A3 V star of AS Eri with a frequency of $59.03 \mathrm{~d}^{-1}$ and second and third oscillation modes with frequencies $62.5 \mathrm{~d}^{-1}$ and $61.67 \mathrm{~d}^{-1}$, respectively. These modes are related to the 5-6 overtone oscillations and are among the shortest period excited in nonmagnetic A-F stars. AS Eri is a very low mass ratio Algol type system. The mass of evolved secondary is only $0.207 M_{\odot}$. The totally eclipsing Algol-type systems S Cnc and DN Ori with A-type primary components and with very low mass evolved secondaries are similar to AS Eri. The A stars in S Cnc, DN Ori and in other very low mass ratio Algol systems may reveal rapid pulsations similar to that observed in the A-type star in AS Eri. Study of the characteristics of rapid pulsations of A-type stars in Algol-type systems (in particular very low mass ratio Algol systems) may enable us to understand the mass-accretion process and evolution of Algol-type close-binary stars.

There are a few peculiar Algol-type systems such as V356 Sgr. It is a 8.9 day period semidetached system consisting of a B4V primary and a giant A2 II secondary. The A2 II secondary has filled its Roche lobe and is now burning hydrogen in a shell. From an analysis of the ultraviolet spectrum of the A2 II star obtained during the total eclipse Polidan (1988) finds that the evolved A2 II component is extremely underabundant in carbon. The cool evolved secondary components in Algol-type systems were also found to show underabundances of carbon (Parthasarathy et al. 1983, Parthasarathy 1990b). Further studies of the A2 II star in V356 Sgr and its pulsation characteristics may enable us to further understand the accretion and evolution of V356 Sgr and similar close-binary stars. High speed photometric monitoring of V 356 Sgr during the totality may reveal the rapid pulsations of the A2 II secondary.

$v$ Sgr, HD 30353 (KS Per), LSS 4300 and CPD-58 2721 (LSS 1922) form a small group of hydrogen poor, helium rich close binaries with A-type components. The primary components in these systems are hydrogen-poor and helium rich late B and A-type 
supergiants. The ultraviolet spectra of $v$ Sgr and HD 30353 indicate that their secondary components may be late evolved OB stars. UV high resolution spectra of these two peculiar binaries with hydrogen-poor A-type supergiants show violet shifted N v, C IV, Si IV, C II, Al III, and Mg II stellar wind profiles (Parthasarathy et al. 1986, 1990) indicating a stellar wind and mass-loss similar to that observed in OB stars. The IRAS far-infrared fluxes also show evidence for the presence of warm and cold circumstellar dust shells (Parthasarathy 1990b). Further studies of $v$ Sgr, HD 30353, LSS 4300 and LS 1992 are needed to understand their peculiar chemical composition, mass-loss rates and evolution. It is also important to search for the rapid pulsations of the A-type primary components of $v$ Sgr and HD 30353 (KS Per).

Another important class of close-binaries some of which contain an evolved A or a Main Sequence A star are planetary nebulae with binary central stars. There are more than 16 planetary nebulae with close-binary nuclei (Bond 2000) with periods of 2.7 hours to 16 days. These are so close that the nebulae must have been ejected through commonenvelope interactions (Bond 2000 and references therein). Further studies of planetary nebulae with binary nuclei with $\mathrm{A}$ and $\mathrm{F}$ type components will enable us to derive accurate distances and luminosities of central stars, as the Bolometric Corrections of A and $\mathrm{F}$ type stars are very small. Also detailed study of such objects may enable us to understand the formation of jets, bipolar structures, shaping and evolution of planetary nebulae, and common envelope evolution (Bond 2000).

\section{Stars related to massive A supergiants}

Massive stars of 8 to $40 M_{\odot}$ during their post-Main Sequence evolution go through the A supergiant stage. For more detail see Przybilla (2005). Some luminous blue variables (LBVs) are related to massive A supergiants.

LBVs include Hubble-Sandage variables, P Cygni stars, and S Doradus stars. All are hot massive stars and show A supergiant type spectra during the periods of maximum brightness. LBVs show variations in brightness by 1 to 2 magnitudes on time scales of a few decades. Occasionally, they erupt and increase in their brightness by more than 3 magnitudes. During the periods of maximum brightness they resemble spectral type A supergiants. For more details on LBVs see Nota \& Lamers (1997a). The LBVs are the very short lived phase of the evolution of very massive stars.

There are about 10 LBVs known in the Milky Way Galaxy, about six in the LMC, about two in the SMC, and four or five in M31 and M33. Some of the well studied LBVs that show A supergiant spectrum during the period of maximum brightness are AG Car, HR Car, S Dor, R 71, and R 127. Most LBVs have nebulae and circumstellar dust shells that are the result of the ongoing large scale mass-loss and outbursts. HR Car is associated with circumstellar filamentary nebula. From a recent set of high resolution coronographic images Notal et al. (1997b) confirm that the nebula around HR Car is truly bipolar and reminiscent of the $\eta$ Carinae Nebula. The polarization measurements of HR Car also indicate bipolar geometry (Parthasarathy et al. 2000c).

Recently Clark \& Negueuela (2004) discovered W 243 in the galactic starburst cluster Westerlund 1 to be a luminous blue variable. They find the spectral type of W 243 to be A2 Ia with a rich emission line spectrum indicating high mass loss rate.

Waters et al. (1997) discuss the solid state features in the ISO-SWS spectra of some LBVs. They find evidence for crystalline forms silicates with a composition similar to those seen in red supergiants, AGB and post-AGB stars. They suggest that the dust was formed in a high-density, probably also high temperature environment, during the red supergiant phase of LBVs or in an ejection during a very red LBV phase. Observationally, 
there is strong evidence that very massive stars do not evolve to become red supergiants (Langer 1993) or if they do they spend a very short time as a yellow or red supergiant. There are few supergiants of this type with circumstellar dust shells. Some authors classified these stars with supergiant spectra as low mass stars in post-AGB stage of evolution while others as massive supergiants. The best known examples are HD 168625 (IRAS 18184-1623) (classified as A-type hypergiant by Morgan et al. 1955 and also as a postAGB A supergiant (Parthasarathy 1989)) and HD 179821 (IRAS 19114+0002) (classified as a massive yellow supergiant (Jura et al. 2001) and also as post-AGB star (Pottasch \& Parthasarathy 1988)).

Sterken et al. (1999) classified HD 168625 as a variable A-type hypergiant or marginally dormant LBV. On the basis of spatially resolved nebula around HD 168625 Hutsemekers et al. (1994) classified it as an LBV. From the velocities and chemical composition derived from spatially resolved spectroscopy Nota et al. (1996) confirm that the nebula is associated with the central star HD 168625 and it is nitrogen rich. It was also classified as a post-AGB candidate on the basis of far-infrared (IRAS) colours and detached cold circumstellar dust shell. Its far IR colours are similar to that of post-AB stars and protoplanetary nebulae (Parthasarathy 1989). Sivarani (2000) (see Garcia Lario et al. 2001) found that the equivalent widths of the spectral lines in the high resolution spectrum of HD 168625 are variable. She also finds that the star is nitrogen rich in agreement with the nitrogen-rich nebula, indicating that the nebula may have been ejected after the star became nitrogen-rich during the CNO cycle. The distance and luminosity derived from the Hipparcos parallax seem to be inconsistent with the previous distance estimates (Nota et al. 1996, Hutsmekers et al. 1994, Sivarani 2000, Garcia-Lario et al. 2001). The evolutionary status of HD 168625 is unclear, it may be a LBV or a post-AGB star (Sivarani 2000 ) with a core mass of the order of 0.8 to $1.0 M_{\odot}$. The overabundance of nitrogen is similar to that observed in type I planetary nebulae.

HD $179821(V=8.4$, G5Ia, b=4.96) (AFGL $2423=$ IRAS $19114+0002)$ continues to be the subject of much debate as to whether it is a nearby (distance $1 \mathrm{kpc}$ ) post-AGB star (Pottasch \& Parthasarathy 1988) or a distant (distance $6 \mathrm{kpc}$ ) high mass $\left(30 M_{\odot}\right)$ postred supergiant near the Humphreys-Davidson limit that will become a supernova within the next 100000 years (Jura et al. 2001). Detailed spectroscopic abundance analyses and atmospheric parameters of HD 179821 (Reddy \& Hrivnak 1999, Thevenin et al. 2000) have failed to resolve the issue. Jura et al. (2001) argue that HD 179821 is a hypergiant at a distance of $6 \mathrm{kpc}$ from the Sun. HD 179821 is a strong IRAS source with 25 micron flux of 650 Jy (Pottasch \& Parthasarathy 1988). The extended dust shell scatters light at optical and near-IR wavelengths (Ueta et al. 2000, Jura \& Werner 1999). Thevenin et al. (2000) on the basis of a LTE analysis of its spectra find $T_{\text {eff }}=5660 \mathrm{~K}, \log g=-1.0$ and $[\mathrm{Fe} / \mathrm{H}]=$ -0.5 and underabundances of carbon and the s-process element zirconium indicating that HD 179821 has not gone through the third dredge-up. They find its abundance pattern to be similar to that of the carbon-poor halo planetary nebula DDDM-1. They conclude that it is a low mass, slightly metal-poor star in the post AGB stage of evolution (Thevenin et al. 2000). However, Jura et al. (2001) on the basis of detailed high resolution mapping of the $\mathrm{J}=1-0 \mathrm{CO}$ emission from the circumstellar shell around HD 179821 conclude that it is a highly evolved massive supergiant and that it may explode as a supernova within the next 100000 years.

If it is a massive star evolved from A-type LBV supergiant phase to the present yellow/red hypergiant phase, significant variations in brightness and spectrum are expected. However there is no observational evidence for any major changes in brightness, colour and temperature and spectral type during the past 100 years (Arkhipova et al. 2001c, Jura et al. 2001). Based on ten years of photometric monitoring of HD 179821, Arkhipova 
et al. (2001c) found that it exhibits semiregular light variations with amplitudes of 0.25 , 0.15, 0.10 magnitudes in the UBV bands respectively. The variability seems to be due to pulsation similar that observed in other post-AGB A and F supergiants. The characteristics of HD 179821 appears to be different from that of the yellow hypergiant star IRC +10420 whose spectral type is F8 Ia, although there are indications of a recent increase in temperature, up to A5 type (Klochkova et al. 1997). Accurate distance determinations in the next one or two decades may enable us to understand the evolutionary status of HD 179821 and HD 168625 and other similar supergiants.

\section{References}

Acker A., Neiner C. 2003 A\& A, 403, 659

Ambika S., Parthasarathy M., Aoki W. 2004 A\&A 417, 293

Ambika S., Parthasarathy M. 2004 Preprint

Arkhipova V.P., Ikonnikova N.P., Noskova R.I. 1999 Ast. Lett. 25, 25

Arkhipova V.P., Klochkova V.G., Sokol G.V. 2001a Ast. Lett. 27, 99

Arkhipova V.P., Ikonnikova N.P., Noskova R.I., et al. 2001b Ast. Lett. 27, 719

Arkhipova V.P., Ikonnikova R.I., Noskova R.I., et al. 2001c Ast. Lett. 27, 156

Ashok N.M., Banerjee D.P.K 2003 A $\mathscr{E} A$ 409, 1007

Banerjee D.P.K., Ashok N.M. 2002 A\&A 395, 161

Banerjee D.P.K., Varricatt W.P., Ashok N.M., et al. 2003 ApJ 598, L31

Blöcker T. 1995 A\&GA 299, 755

Blöcker T., Schönberner D. 1997 A\&A 324, 991

Bobrowsky M., Sahu K.C., Parthasarathy M., Garcia-Lario P. 1998 Nature 392, 469

Bond H.E. 1991 IAU Symposium 145, 341

Bond H.E. 2000 ASP Conf. Ser. 199, 115

Bond H.E. 2003 Nature 422, 405

Bond H.E., Luck R.E. 1987 ApJ 312, 203

Clark J.S., Negueruela I. 2004 A $\& A$ 413, L15

Cohen M., et al. 1975 ApJ 196, 179

Cohen M., Van Winckel H., Bond H.E., et al. 2004 AJ 127, 2362

Conlon E.S., Dufton P.L., Keenan F.P. 1991 MNRAS 248, 820

Conlon E.S., Dufton P.L., McCausland R.J.H., et al. 1993 ApJ 408, 593

Conlon E.S., Dufton P.L., Keenan F.P. 1994 A $\& A$ 290, 897

Davies M.B., Piotto G., de Angeli F. 2004 MNRAS 348, 129

de Boer K.S. 1985 A\&A 142, 321

de Boer K.S. 1987 IAU Colloq. 95, 95

De Marco O., Lanz T., Ouellette A., et al. 2004 ApJ 606, L151

Dorman B., Rood T., O'Connell R.W. 1993 ApJ 419, 596

Duerbeck H.W., Liller W., Sterken C., et al. 2000 AJ 119, 2360

Fiebelman W.A 1995 ApJ 443, 245

Gauba G., Parthasarathy M. 2003 A\&A 407, 1007

Gauba G., Parthasarathy M. $2004 A \mathscr{E} A$ 417, 201

Gauba G., Parthasarathy M., Reddy, B.E. $2004 A \& A$ in press

Garcia-Lario P., Parthasarathy M., De Martino D., et al. 1997 A $\mathscr{E} A$ 326, 1103

Garcia-Lario P., Sivarani M., Parthasarathy M., et al. 2001 in Post-AGB objects, eds. Szczerba Es Gorny Kluwer, 309

Gonzalez G., Wallerstein G. 1992 MNRAS 254, 343

Harris H.C., Nemec J.M., Hesser J. 1983 PASP 95, 256

Harrison T.E. 1996 PASP 108, 1112

Herwig F. 2001 ApJ 554, L71

Hrivnak B.J., Kwok S., Volk K.M. 1989 ApJ 346, 265

Hutsemekers D., Van Drom E., Gosset E. 1994 A $\& A$ 290, 906 
Jasniewicz G., de Laverny P., Parthasarathy M., et al. 2004 A $\& A 423,353$

Iben I.Jr. 1982 ApJ 260, 821

Iben I.Jr., MacDonald J. 1995 White Dwarfs ed. D.Koester \& K.Werner (Springer), p48

Jura M., Werner M.W. 1999 ApJ 525, 113

Jura M., Velusamy T., Werner M.W. 2001 ApJ 556, 408

Klochkova V.G., Chentsov E.L., Panchuk V.E., 1997 MNRAS 292, 19

Klochkova V.G., Yushkin M.V., Miroshnichenko A.S., et al. 2002 A $\& A$ 392, 143

Kwok S. 1993 ARAEA 31, 63

Lamers H.J.G.L.M., Waters L.B.F.M., et al. 1986 A $\& A$ 154, L20

Langer N. 1993 ASP. Conf. Ser. 35, 159

Lawlor T.M., MacDonald J. 2002 ASP Conf. Ser. 279, 193

Mapelli M., Sigurdsson S., Monica C., et al. 2004 Apj 605, L29

Martini P., Wagner R., Tomaney A. $1999 A J$ 118, 1034

Mateo M. 1993 ASP Conf. Ser. 53, 74

McCausland R.J.H., Conlon E.S., Dufton P.L. et al. 1992 ApJ 394, 298

Mkrtichian D.E., Kusakin A.V., Rodriguez E., et al. 2004 A $\& A$ 419, 1015

Moehler S. 2001 PASP 113, 1162

Moehler S. 2005, These Proceedings, 395

Moehler S., Heber U. 1998 A\&̈A 335, 985

Moehler S., Heber M., Lemke M., et al. 1998 A\&SA 339, 537

Moehler S., Sweigart A.V., Landsman W.B., et al. 1999 A\&GA 346, L1

Monier R., Parthasarathy M. 1999 A $\& A$ A 341, 117

Mooney C.J., Rolleston W.R.J., Keenan F.P., et al. 2002 MNRAS 337, 851

Mooney C.J., Rolleston W.R.J., Keenan F.P., et al. $2004 A \mathscr{E} A$ (in press)

Morgan W.W., Code A.D., Whitford A.E. 1955 ApJS 2, 41

Munari U., Henden A., Kiyota S., et al. 2002 A\& $A$ 389, L51

Nota A., Pasquali A., Clampin M., et al. 1996 ApJ 473, 946

Nota A., Lamers H.J.G.L.M 1997a ASP Conf. Ser. 120,

Nota A., Smith L., Pasquali A., et al. 1997b ApJ 486, 338

Parthasarathy M. 1989 IAU Colloquium 106, 384, eds. H.R.Johnson and B.Zuckerman, CUP

Parthasarathy M. 1990a IAU Symp. 145, poster papers: p119, eds. Michaud, Tutukov, Bergevin

Parthasarathy M. 1990b BASI 18, 261

Parthasarathy M. 1993a ApJ 414, L109

Parthasarathy M. 1993b ASP Conf.Ser. 45, 173

Parthasarathy M. 1994 ASP Conf.Ser. 60, 261

Parthasarathy M. 1999 IAU Symp. 191, 475

Parthasarathy M. 2000 IAU Symp. 177, 225

Parthasarathy M., Pottasch S.R. 1986 A $\& A$ 154, L16

Parthasarathy M., Pottasch S.R. 1989 A $\mathscr{G} A$ 225, 521

Parthasarathy M., Lambert D.L., Tomkin J. 1983 MNRAS 203, 1063

Parthasarathy M., Cornachin M., Hack M. 1986 A\& A 166, 237

Parthasarathy M., Hack M., Tektunali G. 1990 A\&A 230, 136

Parthasarathy M., Garcia-Lario P., Pottasch S.R. 1992 A $\& A$ 264, 159

Parthasarathy M., Garcia-Lario P., Pottasch S.R., et al. 1993 A $\& A$ 267, L19

Parthasarathy M., Garcia-Lario P., de Martino D., et al. 1995 A $\& A$ 300, L25

Parthasarathy M., Acker A., Stenholm B. 1998 A\&A 329, L9

Parthasarathy M., Vijapurkar J., Drilling J.S. 2000a $A \& A S$ 145, 269

Parthasarathy M., Garcia-Lario P., Sivarani T., et al. 2000b A\&A 357, 241

Parthasarathy M., Jain S.K., Bhatt H.C 2000c $A \mathscr{E} A$ 355, 221

Parthasarathy M., Garcia-Lario P., Gauba G., et al. 2001a A\&A 376, 941

Parthasarathy M., Gauba G., Fujii T., Nakada Y. 2001b in Post-AGB objects as a phase of stellar evolution, 29, (eds Szczerba \& Gorny :Kluwer)

Piotto G., et al. 2002 A $\&$ A 391, 945

Polidan R.S. 1988 Decade of UV Astronomy with IUE ESA SP-281 Vol 1, 205 
Pottasch S.R., Parthasarathy M. 1988 A $\mathscr{G} A$ 192, 182

Preston G.W., Sneden C. 2000 AJ 120, 1014

Preston G.W., Sneden C. 2005, These Proceedings, 403

Przybilla, N. 2005, These Proceedings, 411

Reddy B.E., Hrivnak B.J. 1999 AJ 117, 1834

Reddy B.E., Parthasarathy M., Gonzalez G., Bakker E.J. 1997 A\&A 328, 331

Reddy B.E., Lambert D.L., Gonzalez G., Yong D. 2002 ApJ 564, 482

Reyniers M. 2002 Ph.d Thesis

Reyniers M., Van Winckel H., Gallino R., Straniero O. 2004 A $\& A$ 417, 269

Ryans R.S.I., Dufton P.L., Mooney C.J., et al. 2003 A\&A 401, 1119

Sahai R. 2001 in Post-AGB objects as a phase of stellar evolution, p 53, (eds. Szczerba \& Gorny: Kluwer)

Sahai R., Bujarrabal V., Zijlstra A. 1999 ApJ 518, L115

Schönberner D. 1979 A\&SA 79, 108

Schönberner D. 1983 ApJ 272, 708

Schultheis M., Parthasarathy M., Omont A., et al. 2002, A\&SA 386, 899

Sivarani T. 2000 Ph.D thesis Bangalore University

Sivarani T., Bonifacio P., Molaro P., et al. 2004 A $\& A$ 413, 1073

Sivarani T., Parthasarathy M., Garcia-Lario P., et al. 1999 A\&AS 137, 505

Sneden C., Preston G.W., Cowan J.J. 2003 ApJ 592, 504

Sterken C., Arentoft T., Duerbeck H.W. 1999 A $\& A$ 349, 532

Takeda Y., Parthasarathy M., Aoki W., et al. 2002 PASJ 54, 765

Thevenin F., Parthasarathy M., Jasniewicz G. 2000 A $\& A$ 359, 138

Turner D.G., Drilling J.S. 1984 PASP 96, 292

Ueta T., Meixner M., Bobrowsky M. 2000 ApJ 528, 861

Van Winckel H. 2003, ARAESA 41, 391

Van Winckel H., Cohen M., Gull T.R. 2002 A\&A 390, 147

Van Winckel H., Mathis J.S., Waelkens C. 1992, Nature 356, 500

Van winckel H., Waelkens C., Waters L.B.F.M. 1995, A\&A 293, L25

Van Winckel H., Waelkens C., Waters L.B.F.M. 2000, IAU Symposium 177, 285

Venn K.A. 1997, ASP Conf. Ser. 120, 95

Vijh U.P., Witt A.N., Gordon K.D. 2004, ApJ 606, L65

Volk K.M., Kwok S. 1989, ApJ 342, 345

Waelkens C., Waters L.B.F.M., Cassatella A., et al. 1987, A\&SA 181, L5

Waelkens C., Van Winckel H., Bogaert E., et al. 1991, A $\mathscr{E} A$ 251, 495

Waelkens C., Van Winckel H., Waters L.B.F.M., et al. 1996, A\&SA 314, L17

Waelkens C., Waters L.B.F.M., Van Winckel H. 1995, ApSS 224, 357

Waters L.B.F.M., Trams N.R., Waelkens C. 1992, A\& A 262, L37

Waters L.B.F.M., Morris P.W., Voors R.H.M., et al. 1997, ASP Conf.Ser. 120, 326

Whitelock P.A., Menzies J.W., Catchpole R.M., et al. et al. 1989, MNRAS 241, 393

Witt A.N., Boroson T.A. 1990, ApJ 355, 182

Witt A.N., Vijh U.P. 2004, ASP Conf.Ser. 309

Zinn R.J., Newell E.B., Gibson J.B. 1972, A\&A, 18, 390 